\title{
Biomarkers for sepsis: past, present and future
}

\author{
Giuseppe Chesi, Natale Vazzana, Claudio Giumelli \\ Department of Internal Medicine, C. Magati Hospital, Scandiano (RE), Italy
}

\begin{abstract}
Sepsis is a complication of severe infection associated with high mortality and open diagnostic issues. Treatment strategies are currently limited and essentially based on prompt recognition, aggressive supportive care and early antibiotic treatment. In the last years, extensive antibiotic use has led to selection, propagation and maintenance of drug-resistant microorganisms. In this context, several biomarkers have been proposed for early identification, etiological definition, risk stratification and improving antibiotic stewardship in septic patient care. Among these molecules, only a few have been translated into clinical practice. In this review, we provided an updated overview of established and developing biomarkers for sepsis, focusing our attention on their pathophysiological profile, advantages, limitations, and appropriate evidence-based use in the management of septic patients.
\end{abstract}

\section{Sepsis: definition and diagnosis}

Depending on the characteristics of the patient, pathogen profile and setting of development, sepsis could be a rapidly life-threatening condition. ${ }^{1} \mathrm{~A}$ high suspicion index is crucial for appropriate investigations and prompt diagnosis and management. Sepsis is a systemic inflammatory response syndrome (SIRS) caused by infection. ${ }^{2}$ Because SIRS is not specific for sepsis and can be caused by several non-infective conditions, extensive research has been performed to identify rapid biomarkers for differentiating sepsis from other causes of SIRS, in order to limit the inappropriate use of broad-spectrum antibiotics and its consequent deleterious effect on bacterial ecosystem. ${ }^{3}$

\section{Early management of severe sepsis and usefulness of biomarkers}

In the presence of high clinical suspicion of sepsis,

Correspondence: Giuseppe Chesi, Department of Internal Medicine, C. Magati Hospital, viale Martiri della Libertà 2, 42019 Scandiano (RE), Italy.

E-mail: chesig@ausl.re.it

Key words: Antibiotics; biomarkers; C-reactive protein; procalcitonin; sepsis.

Received for publication: 6 October 2016.

Accepted for publication: 20 October 2016.

This work is licensed under a Creative Commons Attribution NonCommercial 4.0 License (CC BY-NC 4.0).

CCopyright G. Chesi et al., 2016

Licensee PAGEPress, Italy

Italian Journal of Medicine 2016; 10:301-307

doi:10.4081/itjm.2016.795 especially in the presence of criteria of severe disease or septic shock, early supportive therapy - including aggressive fluid resuscitation to sustain adequate perfusion and prevent organ dysfunction - and empiric antibiotic therapy should be immediately instituted, tailored on the characteristics of the patient and the clinical setting. ${ }^{4}$ In critically ill patients, microbiological tests preferably obtained before antibiotic administration are more important than diagnostic biomarkers as they can guide adjustments to initial therapy. ${ }^{5}$ In these situations, biomarkers may rather have a role for response monitoring and prognosis. ${ }^{6}$ In case of persistent negative results of certain biomarkers, the diagnosis of sepsis should be reconsidered, especially in the absence of significant improvement after appropriate antibiotic treatment. There is also increasing evidence that biomarkers can guide the duration of antibiotic therapy, promoting shorter duration of treatment in some patients. ${ }^{7}$ Moreover, in uncertain and non-critical situations such as probable or possible sepsis, the use of biomarkers may have a crucial role for confirming or excluding the diagnosis, thus avoiding the institution of inappropriate antibiotic treatments. ${ }^{8}$

\section{Biomarkers: appropriate use in the specific clinical setting}

As for other diagnostic tests, biomarkers of sepsis should be always framed within the specific clinical setting of the patient and ordered only if they may provide additional information. An adequate knowledge of their pathophysiological profile - including the release kinetics, causes of false-positive or -negative results, and serum clearance time - is also required for appropriate and focused clinical use. ${ }^{9}$ Without these preconditions, the use of biomarkers could be confounding and misleading. In the present review, we 
critically discuss the role of biomarkers for sepsis diagnosis and risk stratification, focusing our attention on advantages, limitations, clinical evidence of costeffectiveness and future perspective.

\section{Search strategy and selection criteria}

Data for this Review were identified by searches of PubMed, Web of Science, the Cochrane Library, and references from relevant articles with the search terms biomarker, sepsis, procalcitonin, C-reactive protein, and/or antibiotic therapy. Only articles published in English between January 1990, and March 2016, were included. Publications were selected for review based on original research, randomized controlled trials, and meta-analyses and evidence-based reviews.

\section{Searching for biomarkers: one, no one and one hundred thousand}

In the last 10 -years, approximately 170 potential biomarkers for sepsis have been identified and proposed for clinical use, thus underlining the unmet clinical need of practical tools to improve the diagnosis and management of this serious condition. ${ }^{10}$ Nevertheless, none of the currently available or experimental biomarkers fulfils all the characteristics required of an ideal biomarker (Table 1). Among these molecules, only a few are currently used in clinical practice. Many other biomarkers are still in exploratory phases and their investigation also provides interesting insights into the pathophysiological cascade of sepsisrelated SIRS. ${ }^{11}$ In the following section we review the preclinical and clinical development of currently available biomarkers, focusing our attention on their strength and weakness and trying to extrapolate their appropriate use in the clinical setting.

\section{Biomarkers in sepsis: the past}

The history of biomarkers in sepsis can be briefly summarized, as there were only few indicators of in- fection and/or SIRS until the end of the 90's. The clinical suspicion of sepsis or other infections was supported only by nonspecific markers such as the increase in erythrocyte sedimentation rate and neutrophil count. Microbiology culture tests were also less accurate and slower than today. Nevertheless, in past microbiological epidemiology there was only a fraction of the current number of virulent and resistant pathogens that have been selected by excessive and often irrational use of antibiotics, especially within hospital and nursing institutes.

\section{Biomarkers in sepsis: the present}

The currently available sepsis biomarkers are essentially two - C-reactive protein (CRP) and procalcitonin (PCT). These tests are widely - but not universally - used in the clinical practice for improving sepsis diagnosis and monitoring. ${ }^{12}$ Beyond these true biomarkers, several other laboratory tests can be helpful for septic patient management, including blood cell count to evaluate the presence of leukocytosis or leukopenia, and - more important - serum lactate levels as a marker of tissue hypoperfusion. ${ }^{13}$ All these markers can be used synergistically with bedside ultrasonographic evaluation of inferior vena cava diameter and collapsibility index in order to support the initial diagnosis and to estimate adequate fluid support and response monitoring. ${ }^{14}$

\section{C-reactive protein}

CRP has a long history as it was first detected by Tillet and Francis in 1930 in the serum of patients with pneumococcal pneumonia. ${ }^{15}$ It was subsequently recognised as an acute-phase protein and proposed as a clinical marker of inflammation independent of the underlining cause. CRP is a member of the pentraxin family of calcium-dependent ligand-binding plasma proteins. It is synthesized and released by hepatocytes in response to cytokines, particularly interleukin (IL)-6. Its half-life is about $19 \mathrm{~h}$ and it is poorly affected by inter-

Table 1. Characteristics of the ideal biomarker for sepsis and relative clinical implications.

\begin{tabular}{ll}
\hline Characteristic & Clinical implications \\
\hline High sensitivity & Low rate of false-negative results \\
\hline High specificity & Low rate of false-positive results \\
\hline Early release peak & Early diagnosis \\
\hline Reflective of disease severity and course & Risk stratification, treatment decisions and response evaluation \\
\hline Rapid, simple, inexpensive, and standardized assay & Readily available, reproducible and cost-effective \\
\hline Limited renal or hepatic clearance & Useful in patients with sepsis-related organ dysfunctions \\
\hline
\end{tabular}


current pathophysiological circumstances. Following its release induced by specific cytokines, CRP itself acts as a direct inflammatory mediator in response of several pathophysiological triggers. In the clinical setting, the major limit of CRP is the lack of specificity, as it is constantly increased in SIRS, independent of the etiology, as well as in other inflammatory conditions such as disreactive or autoimmune diseases and cancer, thus it cannot be used as a diagnostic marker. ${ }^{16}$ However, due to its short half-life, it may be helpful to monitor the course of certain disorders, including infections. ${ }^{17}$ Some data suggest that CRP may be used for diagnostic purpose in combination with other biomarkers such as PCT. ${ }^{18} \mathrm{Nev}$ ertheless, its positive predictive value for sepsis diagnosis remains limited even if higher threshold levels are chosen. ${ }^{19}$ On the contrary, CRP has a high negative predictive value against sepsis, especially if measured few hours after symptoms onset. ${ }^{20}$

\section{Procalcitonin}

PCT has emerged as the most interesting biomarker for the diagnosis of infective diseases. ${ }^{21}$ In recent years an increasing number of studies have investigated the use of PCT as a biomarker of bacterial disease, focusing on its impact on diagnostic performances and management of patients with several infective conditions..$^{22,23}$ The great interest on this molecule is due to its high specificity for bacterial infections, even though recent data have identified specific conditions associated with false-positive PCT increases..$^{24,25}$ PCT is a 116 amino-acid peptide precursor of the hormone calcitonin and is mainly released by the parafollicular $\mathrm{C}$ cells of the thyroid. Under normal circumstances, virtually all released PCT is cleaved into calcitonin by a specific protease, thus very low or absent levels are detectable in serum. ${ }^{26} \mathrm{PCT}$ is rapidly released by several tissues and inflammatory cells in response to bacterial toxins and bacteria-specific proinflammatory mediators, and its cleavage is simultaneously inhibited; therefore, serum PCT levels may dramatically increase in patients with bacterial infections, thus representing a helpful biomarker for early diagnosis of sepsis in critically ill patients. ${ }^{27} \mathrm{PCT}$ has a favourable kinetics as diagnostic biomarker, with a rapid rise following its induction, and early peak levels (6-8 $\mathrm{h}$ after symptom onset). In addition, PCT shows a rapid decline following treatment or removal of the underlying trigger, with a half-life ranging from 25 to $45 \mathrm{~h}$ and little affected by renal function. Based on this profile, PCT use has been extended from a diagnostic purpose to patient monitoring and response evaluation. PCT levels may also have prognostic implication. In fact, elevated PCT concentrations and PCT nonclearance are strongly associated with all-cause mortality in septic patients. ${ }^{28}$ It should be noted that a profound knowledge of PCT induction mechanisms and kinetics is required for its appropriate use in the clinical setting. For example, PCT determination should be required only after appropriate time - at least 6-8 $\mathrm{h}$ - from symptom onset. Moreover, clinicians should know that PCT peaks are quantitatively related to the degree of bacteremia, thus PCT can be unhelpful in localised or poorly bacteremic infections. Table 2 shows the most common cause of false-positive or false-negative test results. As stated before, patients with severe sepsis or septic shock should receive prompt empirical antibiotic treatment after blood culture has been obtained. Thus, the most appropriate clinical use of PCT is for the diagnosis and management of patients with possible or probable sepsis, particularly in the presence of respiratory tract infections. ${ }^{29}$ In fact, in these non-critical situations, the decision to start antibiotic treatment can be delayed until the results of PCT and/or other biomarkers are available. Table 3 reports the PCT cut-

Table 2. Common causes of false-positive and false-negative procalcitonin test results.

\begin{tabular}{ll}
\hline False-positive results & False-negative results \\
\hline Cancer (e.g., medullary thyroid cancer, SCLC) & Early course of infection \\
\hline Severe SIRS & Previous antibiotic therapy \\
\hline End-stage renal disease & Immunocompromised host \\
\hline Massive stress (trauma, burns, surgery, ARDS) & Localized infection or subacute course
\end{tabular}

Autoimmune disease (e.g., vasculitis, Still disease)

Prolonged, severe cardiogenic shock or organ perfusion abnormalities

Acute coronary syndrome

Malaria and some fungal infections

Drugs (anti-lymphocyte globulins, alemtuzumab, IL-2)

SCLC, small-cell lung cancer; SIRS, systemic inflammatory response syndrome; ARDS, acute respiratory distress syndrome; IL, interleukin. 
off values for clinical decisions in lower respiratory tract infections and critically ill patients. ${ }^{29-32}$ PCT may also be used for response monitoring and decision to discontinue antibiotic treatment. In fact, there is increasing evidence that PCT guidance can reduce the duration of antibiotic treatment for patients with bacterial infection. In the PRORATA trial, which included non-surgical patients in intensive care units, investigators were encouraged to discontinue antibiotics when PCT concentration was less than $80 \%$ of the peak concentration or an absolute concentration of less than $0.5 \mu \mathrm{g} / \mathrm{L}$ was reached. ${ }^{30}$ A significant reduction in antibiotic treatment duration was obtained, but data on mortality were controversial. ${ }^{31}$ Similar results were found in a subsequent meta-analysis of seven studies comprising a total of 1075 patients with severe sepsis or septic shock. More recently, the SAPS trial used the same decisional criterion of PRORATA in the same clinical setting and further confirmed that PCT-guided algorithms can reduce the duration of antibiotic treatment. Moreover, the PCT-guided group had a lower mortality than the standard-of-care group. ${ }^{32}$ Although PCT shows a limited positive predictive value outside of the appropriate clinical setting, its negative predictive value is high, especially if persistently low levels are found in repeated measures, and it may help the clinician to consider conditions other than bacterial infections that cause fever or other symptoms. In conclusion, as recently discussed, PCT is the most important bio- marker of sepsis currently available in the clinical setting but it should be handled with care and only with a profound knowledge of its kinetics and causes of false-positive and false-negative results.

\section{Biomarkers: the future}

At present, PCT and PCR are the only markers of sepsis routinely used in the clinical practice in Italy and in most of the developed countries. A major limit of these biomarkers is their relatively low positive predictive value and specificity. Research has then focused on novel tests with increased specificity, and a number of novel molecules have been identified and proposed for clinical use. However, only a few have the kinetic profile, specificity and diagnostic performances required for translation to the clinical setting. In the following section, we briefly review the characteristics of these molecules.

\section{Cytokine biomarkers}

As cytokines are key players in the inflammatory response, they are candidate biomarkers for sepsis. Among these, tumor necrosis factor (TNF)- $\alpha$, IL-1 $\beta$, and IL- 6 have been tested for potential clinical use. These molecules show an early increase after inflammatory stimulation, rapid clearance and extremely high sensitivity. However, their specificity is too low to be

Table 3. Procalcitonin cut-off levels for clinical decisions.

\begin{tabular}{|c|c|}
\hline Serum PCT level & Interpretation* \\
\hline \multicolumn{2}{|l|}{ Suspected lower respiratory tract infection } \\
\hline $\mathrm{PCT}<0.1 \mathrm{ng} / \mathrm{mL}$ & $\begin{array}{l}\text { Very low likelihood for bacterial infection, antibiotic therapy strongly } \\
\text { discouraged }\end{array}$ \\
\hline PCT $0.1-0.25 \mathrm{ng} / \mathrm{mL}$ & Low likelihood for bacterial infection, antibiotic therapy discouraged \\
\hline PCT $0.25-0.5 \mathrm{ng} / \mathrm{mL}$ & $\begin{array}{l}\text { Intermediate likelihood for bacterial infection, antibiotic therapy should be } \\
\text { considered, based on the stability of the patient's clinical condition }\end{array}$ \\
\hline $\mathrm{PCT} \geq 0.5 \mathrm{ng} / \mathrm{mL}$ & High likelihood for bacterial infection, antibiotic therapy encouraged \\
\hline \multicolumn{2}{|l|}{ Suspected sepsis in critically ill patient } \\
\hline PCT $<0.25 \mathrm{ng} / \mathrm{mL}$ & $\begin{array}{l}\text { Very low likelihood for bacterial infection, antibiotic therapy strongly } \\
\text { discouraged }\end{array}$ \\
\hline PCT $0.25-0.5 \mathrm{ng} / \mathrm{mL}$ & Low likelihood for bacterial infection, antibiotic therapy discouraged \\
\hline PCT $0.5-1 \mathrm{ng} / \mathrm{mL}$ & $\begin{array}{l}\text { Intermediate likelihood for bacterial infection, antibiotic therapy should be } \\
\text { considered, based on the stability of the patient's clinical condition }\end{array}$ \\
\hline $\mathrm{PCT} \geq 1 \mathrm{ng} / \mathrm{mL}$ & High likelihood for bacterial infection, antibiotic therapy strongly encouraged \\
\hline \multicolumn{2}{|l|}{ Response evaluation and duration of antibiotic treatment } \\
\hline PCT decrease $\geq 90 \%$ of its peak value or PCT $\leq 0.25 \mathrm{ng} / \mathrm{mL}$ & Antibiotic strongly encouraged \\
\hline PCT decrease $\geq 80 \%$ of its peak value or PCT $\leq 0.5 \mathrm{ng} / \mathrm{mL}$ & Antibiotic cessation encouraged \\
\hline PCT decrease $<80 \%$ & Antibiotic cessation discouraged \\
\hline PCT stable or increased & $\begin{array}{l}\text { Uncontrolled infection, consider changing or expanding antibiotic coverage or } \\
\text { further diagnostic evaluation; increased risk of mortality }\end{array}$ \\
\hline
\end{tabular}

PCT, procalcitonin. *Excludes situations requiring immediate antibiotic treatment (e.g., septic shock, purulent meningitis). Data from ref. ${ }^{29-32}$ 
useful as diagnostic tool. Levels of some cytokines were correlated with disease severity and development of organ dysfunction, making them potentially useful prognosis predictors. Nevertheless, their use is currently limited to research purpose and a translation to the clinical setting is unlikely in short term.

\section{Cell surface markers and soluble receptors}

Although still experimental and far from clinical application, this group comprises some of the most promising molecules that fulfil almost completely the characteristics required for an ideal biomarker. The biomarkers proposed and preliminarily tested include CD64, soluble triggering receptor expressed on myeloid cells (sTREM)-1, and soluble urokinase-type plasminogen activator receptor (suPAR).

CD64 is a membrane glycoprotein with increased expression on neutrophils in subjects with bacterial infections. CD64 has a relatively high specificity and a strong correlation was found between CD64 expression, positive blood culture and disease severity. Based on a pooled analysis of 13 studies, the sensitivity and specificity for CD64 expression on neutrophils and bacterial infection is $79 \%$ and $91 \%$, respectively. ${ }^{33}$ However, the methodological quality of these studies is relatively low and further investigations are needed.

sTREM-1 is a soluble form of TREM-1, a glycopeptide receptor upregulated on the surface of myeloid cells after bacterial infections. Based on available data, its sensitivity and specificity is comparable to currently used biomarkers, thus a translation in the clinical setting seems unlikely within the next few years. ${ }^{34}$

uPAR is a surface signalling receptor expressed on most leukocytes. Its soluble form (suPAR) is found in plasma and other biological fluids after the cleavage of the membrane receptor triggered inflammatory processes. Its performances as diagnostic biomarkers are limited, with less specificity and positive predictive value than currently used molecules. ${ }^{35}$ However, circulating suPAR levels are significantly related to the severity of the inflammatory response and have superior prognostic power over other commonly used biological markers in sepsis as well as in other inflammatory conditions. ${ }^{36}$

\section{Other biomarkers}

Proadrenomedullin (proADM) is the mid-regional fragment derived from the cleavage of ADM, a 52amino-acid peptide produced by the adrenal medulla. proADM is implicated in the pathogenesis of hypotension associated with severe sepsis. proADM showed an elevated power to predict localized bacterial infection and differentiate sepsis from SIRS in pa- tients with hematologic malignancies. ${ }^{37}$ Even though these data should be confirmed in other clinical setting, proADM appears to be a promising biomarker for sepsis diagnosis that may complement the performance of other markers.

Mirco-RNAs (miRNAs) are a recently discovered class of small, non-coding RNAs that regulate protein levels post-transcriptionally. ${ }^{38}$ miRNAs are remarkably stable in the circulation and have been proposed as diagnostic biomarkers of numerous human conditions such as cancer and cardiovascular disease. Moreover, recent studies showed promising results supporting the role of single miRNAand/or multiple miRNAs panels (miRNA signatures) for diagnosis and prognosis of sepsis. ${ }^{39,40}$

Other biomarkers have several limitations that could limit their development and/or translation into the clinical setting. Among the molecules, the following should be cited: LBP (lipopolysaccharide binding protein), HMGB-1 (high mobility group box 1 protein), MIF (macrophage migration inhibitor factor), and angiopoietin.

\section{Combinations of biomarkers}

Recent studies showed that the limitations of single biomarker could be overcome through an integrated combination of clinical variables and laboratory markers, which appear more likely to be able to guide diagnosis or treatment, or assist in prognostication. Several models and scoring systems have been proposed, but the selection of variables that should be included in such scores remains a critical challenge. The infection probability score - which includes six different variables routinely available in septic patients such as temperature, heart rate, respiratory rate, white blood cell count, C-reactive protein, and Sequential Organ Failure Assessment score - is the most validated, simple and universally accessible, ${ }^{41}$ even though its use should be limited to critical patients. More recently, a bioscore combining neutrophil CD64, PCT and sTREM-1 showed interesting diagnostic performances. ${ }^{42}$ Similar results were obtained through a composite score of PCT, proADM, and TNF- $\alpha .^{43}$ In addition, a high negative predictive value for sepsis was found by combining $\alpha-2$ macroglobulin and PCT. ${ }^{44}$ Though interesting, solid data supporting the routine application of these multimarker approaches are lacking. Therefore, it remains crucial that clinicians treating patients with sepsis have detailed knowledge of the characteristics, advantages and limitations of locally available biomarkers, as well as of their integrated use within appropriate decisional algorithms, flow-charts or scoring systems in order to improve the diagnostic performances, therapeutic appropriateness and patients' outcomes. 


\section{Conclusions}

In the last years, the increased number of high-risk patients as well as the selection and propagation of multidrug-resistant organisms has raised new challenges in the management of sepsis. Advancing age increases the prevalence of frail patients with chronic conditions linked to increased risk of sepsis, which sometimes can be severe and difficult to diagnose. As for other diseases, the approach to patients with suspected sepsis should start from a thorough clinical assessment of disease probability and severity, in order to identify subjects who need aggressive resuscitation and early antibiotic treatment. Along with assessment of organ (dys)function, initial evaluation and risk stratification should include the measurement of inferior vena cava collapsibility index and serum lactate levels. Beside this, extensive researches have been carried out in order to identify biomarkers useful for diagnosis, definition of severity, management, and follow-up of sepsis. To date, although with some limitations, the most validated and clinically informative biomarker is PCT, preferably used in combination with C-reactive protein. These molecules provide useful information for diagnosis and prognosis in several clinical conditions, including patients with mild disease (probable or possible sepsis) as well as those with severe sepsis or septic shock. In addition, solid data support the use of PCT guidance to safely reduce and optimize the duration antibiotic therapy. Many other biomarkers are being investigated and tested in clinical studies. Nevertheless, further studies are needed before their use could be translated into the clinical setting.

\section{References}

1. Levy MM, Dellinger RP, Townsend SR, et al. The Surviving Sepsis Campaign: results of an international guideline-based performance improvement program targeting severe sepsis. Intensive Care Med 2010;36:222-31.

2. Singer M, Deutschman CS, Seymour CW et al. The Third International Consensus Definitions for Sepsis and Septic Shock (Sepsis-3). JAMA 2016;315:801-10.

3. Faix JD. Biomarkers of sepsis. Crit Rev Clin Lab Sci 2013;50:23-6.

4. Dellinger RP, Levy MM, Rhodes A, et al. Surviving sepsis campaign: international guidelines for management of severe sepsis and septic shock: 2012. Crit Care Med 2013;41:580-637.

5. O'Grady NP, Barie PS, Bartlett JG, et al. Guidelines for evaluation of new fever in critically ill adult patients: 2008 update from the American College of Critical Care Medicine and the Infectious Diseases Society of America. Crit Care Med 2008;36:1330-49.

6. Kibe S, Adams K, Barlow G. Diagnostic and prognostic biomarkers of sepsis in critical care. J Antimicrob Chemother 2011;66:33-40.

7. Venkatesh B, Kennedy P, Kruger PS, et al. Changes in serum procalcitonin and C-reactive protein following antimicrobial therapy as a guide to antibiotic duration in the critically ill: a prospective evaluation. Anaesth Intensive Care 2009;37:20-6.

8. Wang H, Yin F, Shen DX, et al. Predictive value of procalcitonin for excluding bloodstream infection: results of a retrospective study and utility of a rapid, quantitative test for procalcitonin. J Int Med Res 2013;41:1671-81.

9. Cho SY, Choi SM. Biomarkers of sepsis. Infect Chemother 2014;46:1-12.

10. Pierrakos C, Vincent JL. Sepsis biomarkers: a review. Crit Care 2010;14:R15.

11. LaRosa SP, Opal SM. Biomarkers: the future. Crit Care Clin 2011;27:407-19.

12. Li HX, Liu ZM, Zhao SJ. Measuring both procalcitonin and C-reactive protein for a diagnosis of sepsis in critically ill patients. J Int Med Res 2014;42:1050-9.

13. Marshall JC, Cook DJ, Christou NV, et al. Multiple organ dysfunction score: a reliable descriptor of a complex clinical outcome. Crit Care Med 1995:1638-52.

14. Kosiak W, Swieton D, Piskunowicz M. Sonographic inferior vena cava/aorta diameter index, a new approach to the body fluid status assessment in children and young adults in emergency ultrasound-preliminary study. Am J Emerg Med 2008;26:320-5.

15. Póvoa P. C-reactive protein: a valuable marker of sepsis. Intensive Care Med 2002;28:235-43.

16. Vincent JL, Donadello K, Schmit X. Biomarkers in the critically ill patient: C-reactive protein. Crit Care Clin 2011;27:241-51.

17. Póvoa P, Teixeira-Pinto AM, Carneiro AH; Portuguese Community-Acquired Sepsis Study Group SACiUCI. C-reactive protein, an early marker of community-acquired sepsis resolution: a multi-center prospective observational study. Crit Care 2011;15:R169.

18. Castelli GP, Pognani C, Meisner M, et al. Procalcitonin and $\mathrm{C}$-reactive protein during systemic inflammatory response syndrome, sepsis and organ dysfunction. Crit Care 2004;8:R234-42.

19. Keshet R, Boursi B, Maoz R, et al. Diagnostic and prognostic significance of serum C-reactive protein levels in patients admitted to the department of medicine. Am J Med Sci 2009;337:248-55.

20. Simon L, Gauvin F, Amre DK, et al. Serum procalcitonin and C-reactive protein levels as markers of bacterial infection: a systematic review and meta-analysis. Clin Infect Dis 2004;15;39:206-17.

21. Schuetz P, Albrich W, Mueller B. Procalcitonin for diagnosis of infection and guide to antibiotic decisions: past, present and future. BMC Med 2011;9:107.

22. Riedel S, Melendez JH, An AT, et al. Procalcitonin as a marker for the detection of bacteremia and sepsis in the emergency department. Am J Clin Pathol 2011;135:182-9.

23. Becker KL, Snider R, Nylen ES. Procalcitonin assay in systemic inflammation, infection, and sepsis: clinical utility and limitations. Crit Care Med 2008;36:941-52.

24. Avrillon V, Locatelli-Sanchez M, Folliet L, et al. Lung cancer may increase serum procalcitonin level. Infect Disord Drug Targets 2015;15:57-63.

25. Müller B, Christ-Crain M, Nylen ES, et al. Limits to the use of the procalcitonin level as a diagnostic marker. Clin Infect Dis 2004;39:1867-8.

26. Reinhart K, Meisner M. Biomarkers in the critically ill 
patient: procalcitonin. Crit Care Clin 2011;27:253-63.

27. Wacker C, Prkno A, Brunkhorst FM, Schlattmann P. Procalcitonin as a diagnostic marker for sepsis: a systematic review and meta-analysis. Lancet Infect Dis 2013;13:426-35.

28. Liu D, Su L, Han G, et al. Prognostic value of procalcitonin in adult patients with sepsis: a systematic review and meta-analysis. PLoS One 2015;10:e129450.

29. Schuetz P, Amin DN, Greenwald JL. Role of procalcitonin in managing adult patients with respiratory tract infections. Chest 2012;141:1063-73.

30. Bouadma L, Luyt CE, Tubach F, et al. Use of procalcitonin to reduce patients' exposure to antibiotics in intensive care units (PRORATA trial): a multicentrerandomised controlled trial. Lancet 2010;375:463-74.

31. Prkno A, Wacker C, Brunkhorst FM, Schlattmann P. Procalcitonin-guided therapy in intensive care unit patients with severe sepsis and septic shock-a systematic review and meta-analysis. Crit Care 2013;17:R291.

32. De Jong E, van Oers JA, Beishuizen A. Efficacy and safety of procalcitonin guidance in reducing the duration of antibiotic treatment in critically ill patients: a randomised, controlled, open-label trial. Lancet Infect Dis 2016;16:819-27.

33. Cid J, Aguinaco R, Sánchez R, et al. Neutrophil CD64 expression as marker of bacterial infection: a systematic review and meta-analysis. J Infect 2010;60:313-9.

34. Wu Y, Wang F, Fan X, et al. Accuracy of plasma sTREM-1 for sepsis diagnosis in systemic inflammatory patients: a systematic review and meta-analysis. Crit Care 2012;16:R229.

35. Donadello K, Scolletta S, Taccone FS, et al. Soluble urokinase-type plasminogen activator receptor as a prognostic biomarker in critically ill patients. J Crit Care 2014;29:144-9.
36. Backes Y, van der Sluijs KF, Mackie DP. Usefulness of suPAR as a biological marker in patients with systemic inflammation or infection: a systematic review. Intensive Care Med 2012;38:1418-28.

37. Al Shuaibi M, Bahu RR, Chaftari AM, et al. Proadrenomedullin as a novel biomarker for predicting infections and response to antimicrobials in febrile patients with hematologic malignancies. Clin Infect Dis 2013;56:943-50.

38. Cortez MA, Bueso-Ramos C, Ferdin J, et al. MicroRNAs in body fluids - the mix of hormones and biomarkers. Nat Rev Clin Oncol 2011;8:467-77.

39. Wang H, Meng K, Chen WJ, et al. Serum miR-574-5p: a prognostic predictor of sepsis patients. Shock 2012;37:263-7.

40. Wang H, Zhang P, Chen W, et al. Serum microRNA signatures identified by Solexa sequencing predict sepsis patients' mortality: a prospective observational study. PloS One 2012;7:e38885.

41. Peres Bota D, Mélot C, Lopes Ferreira F, Vincent JL. Infection probability score (IPS): A method to help assess the probability of infection in critically ill patients. Crit Care Med 2003;31:2579-84.

42. Gibot S, Béné MC, Noel R, et al. Combination biomarkers to diagnose sepsis in the critically ill patient. Am J Respir Crit Care Med 2012;186:65-71.

43. Angeletti S, Dicuonzo G, Fioravanti M, et al. Procalcitonin, MR-proadrenomedullin, and cytokines measurement in sepsis diagnosis: advantages from test combination. Dis Markers 2015;2015:951532.

44. Kelly BJ, Lautenbach E, Nachamkin I, et al. Combined biomarkers discriminate a low likelihood of bacterial infection among surgical intensive care unit patients with suspected sepsis. Diagn Microbiol Infect Dis 2016; 85:109-15. 